\title{
Cycles Generated by the Consumer Durables Sector
}

\author{
Péter Mihályi ${ }^{1,2}$, Ádám Zlatniczki ${ }^{3 *}$, András Telcs ${ }^{3,4}$ \\ ${ }^{1}$ Corvinus University of Budapest, 1093 Budapest, Fővám tér 8, Hungary \\ ${ }^{2}$ Central European University, 1051 Budapest, Nádor u. 9, Hungary \\ ${ }_{3}$ Department of Computer Sciences and Information Theory, Faculty of Electrical Engineering and Informatics, \\ Budapest University of Technology and Economics, H-1521 Budapest, P.O.B. 91, Hungary \\ ${ }^{4}$ University of Pannonia, 8200 Veszprém, Egyetem u. 10, Hungary \\ *Corresponding author, email: adam.zlatniczki@cs.bme.hu
}

Received: 17 July 2017, Accepted: 27 March 2018, Published online: 24 April 2018

\begin{abstract}
We conjecture that in highly developed economies business cycles show positive feedback mechanism in which the households amplify the crises by refraining to replace old durable goods to new ones. On the one hand, families do not suffer much if they stop buying new consumer durables, such as automobiles, the example we use in this paper, because it is perfectly rational for them to continue to drive their "old" vehicle for quite a while without much welfare loss. On the other hand, families drawing salary from the given sector do suffer from the decline of the demand and if the crisis is deep and the sector has strong influence on the entire economy, all households are effected at the end of the day. In the framework of a two-sector economy agent-based model, we illustrate our thesis with the fluctuations in US car sales in the period 2006-2014. Subsequently we use an agent-based simulation and show that our conjectures do hold if applied to real-life statistical data.
\end{abstract}

\section{Keywords}

business cycle, consumer durables, market shock, agent-based simulation

\section{Downturns with little pain for consumers}

In modern and rich economies the fluctuations in the purchases of consumer durables are rather large, as households change their buying habits in time. This, in turn, is one of the drivers of the business cycles (the relevancy of this factor is a debated point in the business cycle literature for decades, see e.g. Baxter [1] and Molnar [2, 3], Berger and Vavra [4] with diverging conclusions). We will argue that the extraordinary length of the post-2008 recession and the weak recovery after that can, to a large extent, be explained by the fact that consumers didn't suffer much when they stopped buying cars. We put three plausible assumptions into the focus of the present paper to yield a simplified market model.

(1) Virtually all households possess a broad palette of long-lasting consumer durables (CD), such as cars, televisions, refrigerators, or furniture. Therefore, the majority of purchases are replacement transactions. An old CD is replaced by a new one, which is somewhat better, bigger or more powerful than the old one.

(2) The majority of households modify their patterns of buying $\mathrm{CD}$-s along the business cycle in response to their own perceived income situation, which is in turn closely correlated with the status of the job market. Urgent replacements arising from a change in family status or a complete breakdown of the existing CD can also happen, but such transactions are in a minority. If the income expectations of the households are positive, they are replacing their consumer durables according to an optimal financial strategy. Cars are the best example. Let us assume that under normal circumstances, the majority of consumers replace their motor vehicles after three years, because in the first three years maintenance costs are low and they can still fetch a relatively good price for the used car when they sell it. However, under the pressure of growing unemployment or merely the fear from it, households can decide to postpone the replacement and continue to use their cars for a fourth or a fifth year with a small financial loss.

(3) Households can pursue such a strategy, because the postponement of the replacement of an amortized, less impressive consumer good is almost painless. In terms of use value, there is not much difference between an identical 3-year old or a 4-year old model. This is corroborated 
by the distinguishing feature of cars, namely their potential for resale [5]. Simply stated, if a used car has real value for the second owner, the first owner might also continue to use it for an extended period without much welfare loss. This is in stark contrast, if compared to standard consumer goods and services (e.g. food or electricity), where the postponement of purchase means an immediate and proportionate reduction of consumer utility and welfare. Another aspect is that many durable goods are considered status symbols, therefore, imply conspicuous consumption, which is easier to reduce [6].

While the consumers can, as we specified above, almost painlessly reduce their automobile purchases, this drop of demand will be immediately translated into a $33 \%$ drop in car sales (if we continue to use the same example) already in the first year and an even bigger one in the second year. In other words, as consumers the households do not suffer much from the cyclical nature of their car purchases, but they are badly hurt if the breadwinners of the households concerned are directly employed in the automobile sector. The falling sales of cars leads to rising unemployment and a rise of the fear from unemployment, and therefore a secondary reduction in new car purchases by the affected workers and those who are already afraid of being affected later. This is a classical positive feedback mechanism - the necessary condition of a cycle. The markets of other consumer durables, like televisions, refrigerators, furniture, etc. work in the same way, although the time of their wearand-tear is usually longer.

The postponement, of course, cannot be extended into infinity. After five or more years cars must be replaced for all sorts of technical and financial reasons. Households will be more and more motivated to buy a new car, even if this would require a reduction of the level of their customary consumer goods and services purchases. As the car sales start to increase, it will lead to an improvement on the labor market, which in turn will lead to higher incomes and a more optimistic assessment of the future. This is, once again, the appearance of the positive feedback mechanism.

In this paper we present a simple model of a two-sector economy. We derive the equilibrium state of the economy and show exponential convergence to it. This exponential convergence means that the change of the behavior of the consumer results in the collapse of the sector of consumer durables. An oversimplified model is analytically tractable, but a more realistic is not. Hence a multi-agent simulation is developed on a bit more sophisticated, hence more realistic model. Simulations are in full agreement with the claims of the analysis and fit well to the real life data.

\section{The simulation model 2.1 A simplified picture}

We consider a closed, two-sector economy. The first one produces ordinary consumer goods and services (CG), the second produces consumer durables (CD). The producers operate separately from each other on the two markets and don't hold stocks. Labor is not moving between the sectors. We assume that each consumer-household is a single unit and all are uniform in their (possibly random) behavior and initial wealth. The model treats time as a discrete variable. A part of the households' income is spent on CG-s and CD-s, while a certain part is saved. In each time period consumers purchase a fixed amount of CG-s, consume them immediately, and may or may not buy CD-s. The consumers are maximizing their utility arising from consumption. Since their CG consumption is held constant, the variation of their utility comes entirely from the variation of their CD stock and spending. CD-s are depreciated with an objectively definable fixed rate $\mathrm{q}$ in each period, but it's influence depends on the consumers utility perception determined by their expectations fluctuating along the business cycle.

The quantitative description of the above model boils down to a simple dynamic determined by a two-by-two matrix. If we impose natural restrictions on some parameters, like depreciation rate, inflation, consumption rate, the model converges toward an equilibrium state. With elementary matrix algebra it can be shown that if the economy started from a state different from the equilibrium, it converges to it with exponential speed. On the other hand, if an external effect changes (lowers) the level of the purchasing power/intention in the economy, it responds with a sharp (exponentially fast) drop of the production and need of labor - i.e. toward a lower level equilibrium. The exponential speed of the change is the consequence of the multiplicative nature of the feedback loop between the labor need and demand for $\mathrm{CD}$-s. If the expectations of households are optimistic, these households replace their durables with the amortization rate q, if bad, households will replace their CD-s less frequently. The decline towards the new lower level equilibrium is exponentially fast. If the better expectations return the recovery is, at least theoretically, again exponential. However, we do not present the analytical resolution of our model. It needs more and cumbersome notations, while the calculation is elementary 
linear algebra and the result is perfectly matching the one we get from agent-based models. On the other hand, as we bring our model closer to reality, adding further components to it, the extended model becomes intractable analytically, yet agent-based models are still able to handle that.

Of course, in the reality we never see such rapid recovery. While people's negative expectations trigger drastic drop of demand on the CD market, demand is not bouncing back in a single period. The situation improves only gradually and the recovery of the economy is slower than exponential. It might require more than 3-5 years, even if we narrow our investigation only to the car market. ${ }^{1}$ Such asymmetry is also clearly recognizable and well-researched in stock exchange price changes. One may incorporate such personal and random effects into our model, but the mathematical treatment would be less appealing. Instead, we build a multi-agent simulation which can host a much richer scenario, several further parameters, utility functions and market effects, than the oversimplified model described above.

\subsection{Mathematical models and their relationship to multi-agent simulation}

Mathematical models are widely used around the world as the main reference for forecasting purposes. They can be grouped into static, dynamic and stochastic dynamic models. A static model consists of a set of equations with all the endogenous and exogenous variables referring to the same time period. Dynamic models specify endogenous variables as functions of lagged endogenous variables, exogenous variables and exogenous shocks. Depending on the model parameters the values of the endogenous variables might converge to (or oscillate around) a steadystate or explode. The changes in the exogenous variables might have different effects given they are transitory or permanent. Even the response of an endogenous variable to the change of an exogenous variable might have different components: the initial effect of the change (the impact multiplier), the response over time (the dynamic multiplier) and the aggregate response over time (the total

1 According to official US data, total light weight vehicle sales (autos \& light trucks) started to fall before the general recession of the economy. In mid-2005, the peak of annualized monthly sales figures was above 21 million units, which more than halved - i.e. it fell below to 9.2 million - by February 2009. According to the annual 2014 and 2015 data, sales climbed up to 16.8 million and 17.8 million respectively. Thus, they remained below the 2009 values. http://www.bea.gov/national/xls/ gap_hist.xlsx Table 6. (downloaded on 7 February, 2016). long-run multiplier) - all of these increase the complexity of an analytical solution. Dynamic models can be further expanded into stochastic models, in which the behavior of the endogenous variables cannot be fully explained deterministically, thus random variables following a specified probability distribution are used to include random perturbations into the model [7]. Prescott [8] viewed stochastic models as a paradigm to macroeconomic analysis, analogous to the supply-demand construct of price theory.

A serious problem with stochastic models arises when they have to be solved in order to produce predictions. Fiaschi and Lavezzi [9] find that nonlinear transition paths to steady-states describe the trajectory better than the canonical theoretical models. Under general conditions, nonlinear stochastic models lack an analytical solution and the implications of the models regarding the behavior of the variables, their co-movements or their responses to exogenous shocks [7]. Despite their wide popularity, stochastic models do not have an agreed procedure for solving them - although under specific circumstances analytical solutions exist, as shown by Campbell [10], Marsiglio and La Torre [11], and Bucci et al. [12]. Noy and Nualsri [13] also argue that empirically describing the typical dynamic response of the economy to an exogenous shock makes many of the models (with known analytical solutions) falsifiable.

These stochastic nonlinear models can be (approximately) solved with numerical methods by running computer simulations. Several researchers have started to use multi-agent simulations (also known as multi-agent systems, MAS, or agent-based modelling, ABM) e.g. Bousquet and Le Page [14]. Helbing and Balietti [15] argue that, to some extent, computer simulation can be seen as an experimental technique for hypothesis testing and they can naturally complement classical research methods. Some authors consider MAS as non-transparent or unreliable, but they show that simulations can deliver reliable results even beyond analytical tractability if done properly. Gatti et al. [16] go even further: they argue that macro-level systems do not necessarily inherit their properties from the balance of income and substitution effect that characterizes the preferences of a representative agent; rather they propose that the MAS paradigm should be favored instead of the current dynamic stochastic general equilibrium models. Scientific analysis is often restricted to unrealistic models because of complexity issues, which is not the case for MAS. Simulations are widely used in natural sciences, but they are based on (differential) equations describing 
the change in variables. Such approach is hard to transfer to social sciences, since the system behavior is usually not mathematically formalized. Structural economic models on the other hand have the advantage of being able to be specified by much simpler decision rules on the microlevel of heterogeneous individuals in the economy, policy rules, and identities, rather than complex equations. Even very complex nonlinear systems might become describable in a more understandable way by using much simpler nonlinear decision rules [8]. These behavioral rules are state conditioned, that is, the individuals co-adapt their behavior; hence, predatory and cooperative associations are permitted along with the usual price-quantity relationships, therefore the modelled economies can exhibit self-organization in an easily observable way [17].

This way it is enough to define simple rules which describe how individuals interact with each other and the environment, leading to a very flexible modelling approach. In MAS the individuals of an economy are represented by agents - the local decision of the agents at the micro-level have a complex aggregated effect, which emerges at the macro level and impels the global system [18]. The numerical results generated by MAS need to be analyzed with statistical methods. An exploratory analysis should be done which involves the meaningful individual and aggregate variables. It has to be decided whether model performance has to be analyzed in the equilibrium, out of the equilibrium or both. Since the aggregated macro-function of the decisions is not available as a formula that describes the model behavior, the main goal is to identify it. Another key part is the calibration of the model - the result of the calibration is a parameter set which maximizes model behavior accordance with the real-world system [19].

The appropriate concept to analyze long-run relationships between endogenous and exogenous variables in the presence of a shock is by analyzing their values in their steady-state (market equilibrium) before and after a shock. A steady-state can be obtained by excluding the exogenous shocks (or in the case of a post-shock system, any further shocks) and assuming constant exogenous variables throughout the simulation. By simulating dynamic models (which describe the whole transition process) the trajectory followed by the endogenous variables between their old and new steady-state can be fully described including both short- and long-term effects. This way not just the duration of the transition, but other primary properties (like the evolution of the rate of growth of the output in time) and all the effects throughout the transition can be characterized. If the stochastic process included in the model is stationary than it is mean-reverting, meaning the time-series generated by simulation converges to its mean as time tends to infinity, and anytime a shock is induced it is going to be corrected. Depending on the effect of the shock this may lead to two outcomes: if the shock has transitory effects the process will return to its original mean, or in the case of permanent effects the process will converge to a new mean. Given enough number of simulation iterations the process mean can be identified, which is going to be around the expected value of the model output.

\subsection{Model definition}

\subsubsection{General assumptions}

A market is assumed with two sectors, one that produces consumption goods (denoted $C G S$ ) and another one that produces durable goods (denoted $C D S$ ). The agents that get assigned to the consumption goods sector are denoted by the set $A_{C G S}$, while the ones assigned to the durable goods sector are denoted by the set $A_{C D S}$. We assume that one agent can only be assigned to one of the two sectors. Labor is defined as the number of agents in the sectors - $L_{C G S}$ denotes the total labor in the consumption goods sector, while $L_{C D S}$ denotes the total labor in the consumer durables sector. They are defined as

$L_{C G S}=\left|A_{C G S}\right|$, and

$L_{C D S}=\left|A_{C D S}\right|$,

where $|S|$ is the cardinality of $S$ if $S$ is a set, otherwise it is the absolute value of the scalar $S$. There are two disjoint subsets in each sector: active and passive labor. Agents that are currently employed at time $t$ belong to the active group (denoted with $A$ in the upper index), while the unemployed ones belong to the passive group (denoted with $P$ in the upper index). The number of agents in each sector can be split and defined similarly:

$$
\begin{aligned}
& L_{C G S}^{A}(t)=\left|A_{C G S}^{A}(t)\right|, \\
& L_{C G S}^{P}(t)=\left|A_{C G S}^{P}(t)\right|, \\
& L_{C D S}^{A}(t)=\left|A_{C D S}^{A}(t)\right|, \text { and } \\
& L_{C D S}^{P}(t)=\left|A_{C D S}^{P}(t)\right| .
\end{aligned}
$$

Two product types are defined: consumer goods (CG) which are consumed in one cycle and cars. CG-s are produced by the consumption goods sector while cars are 
produced by the durable goods sector. Each product has price $(P)$, depreciation rate $(\delta)$ and labor required for unit production $(l)$. The price of a car $\left(P_{c a r}\right)$, the price of a CG $\left(P_{C G}\right)$, the depreciation rate of a car $\left(\delta_{c a r}\right)$, and the labor required for producing one car $\left(l_{c a r}\right)$ are model parameters. The depreciation rate of CG-s $\left(\delta_{C G}\right)$ is assumed to be one - this way CG-s increase utility for one period only, stacking them does not result in extra utility. The labor required for unit production of CG-s is defined as

$l_{C G}=\frac{L_{C G S}}{L_{C G S}+L_{C D S}}$.

By specifying $l_{C G}$ this way we guarantee that every agent in the consumption goods sector is employed at any time, since the amount of CG-s required in each period becomes fixed, $L_{C G S}+L_{C D S}$. The increase of per-capita capital is denoted by $\Delta k$. The model assumes that unemployed agents receive either some kind of unemployment benefit in each period, which is enough only to buy the necessary CG-s to sustain themselves, or a (sector specific) wage. The wealth (or capital) of agent $i$ at time $t$ is denoted by $k_{i}(t)$. Klass et al. [20] found that the wealth of the agents is supposed to follow a Pareto distribution,

$k_{i}(0) \sim \operatorname{Pareto}\left(\alpha, x_{m}\right)$,

where $\alpha=1.49$ is the shape and $x_{m}$ is the scale parameter. Based on surveys of the Federal Reserve (available at http:// www.federalreserve.gov/econresdata/scf/scfindex.htm) $x_{m} \approx 87,000[\$]$ is an acceptable choice to provide initial conditions on agent wealth. The agents also have a car at the start - the age of the cars is drawn from a uniform distribution where the minimum is 0 and the maximum is $\left[\delta_{c a r}^{-1}\right]$.

\subsubsection{Agent decision}

In each cycle the agents face a decision problem - they have to decide whether they buy consumer goods, and/or whether they replace their car, given their current preferences. We apply a discrete choice model to grasp the households' spending behavior with two decision variables, $d_{C G S}(t)$ and $d_{c a r}(t)$, which are binary and determine whether they buy or not in the $t^{\text {th }}$ cycle, formally

$d_{C G}(t), d_{\text {replace }}(t) \in\{0,1\}$.

Discrete choice models require the utility of decisions to be specified. We suppose that capital by itself does not have utility, only if it is converted to CG-s or a car. Since the highest priority of a household is self-sustainment the utility of buying CG-s is always maximal, thus $d_{C G}(t)=1$ is constant for any $t$. The utility of replacing an old car (denoted by $U_{\text {replace }}$ ) depends on the time passed since an old car's purchase - as time goes by the utility of the old one decreases gradually. We define the discrete choice at time $t$ as

$d_{\text {replace }}(t)=\left\{\begin{array}{ll}1, & U_{\text {replace }}(t)=1 \\ 0, & U_{\text {replace }}(t)<1\end{array}\right.$.

The utility of a car could be described in many ways - we analyze the effects of applying a quasilinear and an exponential utility function. We construct the utility functions based on the natural depreciation of cars. If we assume linear depreciation the value of a car at time $t$ is

$v(\Delta t)=\max \{P[1-\Delta t \delta], 0\}$,

given $\Delta t$ time passed since its purchase for price $P$ and the depreciation rate is $\delta$. To normalize the value of the car to the $[0,1]$ interval we can divide by $P$, leading to

$\bar{v}(\Delta t)=\max \{1-\Delta t \delta, 0\}$.

The normalization step is involved to avoid problems caused by how numbers are represented by computers, e.g. two different but very small values in an exponent could become equally zero otherwise (there is a similar issue with large exponents as well). Since only the values of different cars are analyzed the normalization does not bias utility-based comparison between products. The value of the car is the function of $\Delta t$ only, and the utility of the car can be expressed by $\Delta t$ as well. Based on the works of Benito [21] and Guiso et al. [22] an increase in risk aversion causes delay in purchase (slower decrease in utility).

If risk aversion is denoted by $a$ it can be incorporated into the relative value of the $\operatorname{car}(\tilde{V})$ as

$$
\tilde{v}(\Delta t, a)=\max \left\{1-\Delta t \frac{\delta}{a}, 0\right\} \text {. }
$$

The intuitive understanding of the relative value is that a household values a used car differently than its depreciation (which is a constant) would suggest, and this difference is conditional on the risk aversion of the household. This way an increase in risk aversion results in a slower decrease in value in the household's point of view.

\subsubsection{Quasilinear utility}

The utility of buying a new car can be expressed in a quasilinear form with the help of the relative value of the existing car. Quasilinear utility functions are defined as $U\left(x_{1}, x_{2}, \ldots x_{n}\right)=x_{1}+\theta\left(x_{2}, x_{3}, \ldots x_{n}\right)$, 
where $x_{i}$ represent the items of a bundle of goods and $\theta(\cdot)$ is a function, see Varian [23]. In our case there are only two items, new and used cars, which can be represented by their $\Delta t$. For new cars $\tilde{v}=1$ always (due to $\Delta t=0$ ). By choosing $\theta(x)=-x$ we get the quasilinear utility function

$U_{\text {replace }}(\tilde{v}(0, a), \tilde{v}(\Delta t, a))=1-\max \left\{1-\Delta t \frac{\delta}{a}, 0\right\}$.

Setting $\theta(x)=-x$ can be interpreted as saying that we are interested in the utility gain by replacing an old car with a new one. An increase in risk aversion causes a slower increase in utility, thus delays purchase given the aforementioned discrete choice. Note that this kind of utility function obeys the law of diminishing marginal utility, as the utility of buying a new car converges to 1 .

\subsubsection{Exponential utility}

Besides the quasilinear, the exponential utility function is a common choice as well, which is defined as

$U(W)=1-e^{-W}$,

where $W$ denotes wealth, as described in the works of Arrow [24] and Pratt [25]. We define the wealth of a household as the value of its car (with the common assumption that capital does not have utility, only consumption). We suppose that a new car represents the highest wealth - as such, its utility is the highest possible, which is 1 for this kind of exponential utility functions, formally

$U\left(W_{\max }\right)=1$.

The utility gained from replacing an existing car is the difference between the utility of a new car and the utility of an existing car:

$U_{\text {replace }}\left(W_{\text {old }}\right)=U\left(W_{\max }\right)-U\left(W_{\text {old }}\right)$.

Combining the last two equations yields

$U_{\text {replace }}\left(W_{\text {old }}\right)=1-U\left(W_{\text {old }}\right)=1-\left(1-e^{-W_{\text {old }}}\right)=e^{-W_{\text {old }}}$,

which leads to the final form

$U_{\text {replace }}(\tilde{v}(\Delta t, a))=e^{-\tilde{v}(\Delta t, a)}=e^{-\max \left\{1-\Delta t \frac{\delta}{a}, 0\right\}}$.

By defining the discrete choice of the agents this way they are fully maximizing their utility function, and extending the use of a consumer durable like automobiles beyond the customary norm (average length of ownership) and delaying the replacement is much less painful than postponing the purchase of, for example, housing services, health or education services etc. The risk aversion coefficient should vary from agent to agent, and it is also a function of time. Initially it is set uniformly as

$a_{i}(0) \sim U\left(a_{\text {lower }}, a_{\text {upper }}\right)$,

where $a_{\text {lower }}$ and $a_{\text {upper }}$ are model parameters. Generally the risk aversion coefficient of an agent does not change without the presence of an exogenous effect (e.g. without a shock), formally

$a_{i}(t)=a_{i}(t-1)$.

\subsubsection{Demand, production and wealth}

After an agent finished re-evaluating its stocks it optimizes its utility. At the start of the period the agent receives unemployment benefit or payment for its work in the preceding period. The capital of agent $i$ in period $t$, denoted $k_{i}(t)$, is calculated as

$k_{i}(t)=\left\{\begin{array}{cc}k_{i}(t-1)+\Delta k_{C G S}, & i \in A_{C G S}^{A}(t) \\ k_{i}(t-1)+\Delta k_{C D S}, & i \in A_{C D S}^{A}(t) . \\ k_{i}(t-1)+\Delta k_{U}, & \text { otherwise }\end{array}\right.$.

Prices are fixed and we disregard relative wage differences and possible changes in real wages - this is the nature of the modelling exercise. As we wrote at the beginning of the paper, we limit our starting conditions to three plausible assumptions.

The consumption of CG by agent $i$ at time $t$, denoted $c_{i}^{C G}(t)$, is given by the agent's decision as

$c_{i}^{C G}(t)=d_{i}^{C G}=1$.

In other words, the agents keep their CG purchase constant. The consumption of cars by agent $i$ in the $t$ cycle is the minimum of the agent's decision (which is zero or one) and the number of new cars it can afford (which is at least zero):

$c_{i}^{c a r}(t)=\min \left\{d_{i}^{c a r}(t), \frac{k_{i}(t)-P_{C G}}{P_{c a r}}\right\}$.

When every agent finishes optimizing its utility the sectors aggregate the agents' consumption to determine the production, $Y(t)$, in the current business cycle. The aggregate consumption is calculated for each sector as

$C_{C G S}(t)=\sum_{\forall i} c_{i}^{C G}(t)$ and

$C_{C D S}(t)=\sum_{\forall i} c_{i}^{c a r}(t)$. 
The maximum possible production in the sectors is

$Y_{C G S}^{\max }=\frac{L_{C G S}}{L_{C G}}$ and

$Y_{C D S}^{\max }=\frac{L_{C D S}}{L_{c a r}}$.

The actual production of sector $s \in\{\mathrm{CGS}, \mathrm{CDS}\}$ at time $t$ is then

$Y_{s}(t)=\min \left\{C_{s}(t), Y_{s}^{\max }\right\}$.

After the production is calculated labor must be redistributed in every sector. The number of agents required for the production in sector $s$ to satisfy consumption is

$\bar{L}_{s}(t)=Y_{s}(t) \times L_{p}$.

The number of agents that will actually be involved in the production is

$\hat{L}_{s}(t)=\min \left\{\bar{L}_{s}(t), L_{s}\right\}$.

If $\hat{L}_{s}(t)>L_{s}^{A}(t)$, then agents have to be hired from the pool of the unemployed. The number of agents that have to be hired is

$h_{s}(t)=\hat{L}_{s}(t)-L_{s}^{A}(t)$.

The new labor is hired from the pool of the unemployed randomly by a uniform distribution. In case $\hat{L}_{s}(t)<L_{s}^{A}(t)$ then employed agents have to be fired (again, randomly with a uniform distribution). The aggregate consumption might exceed the maximum possible production - in such cases the agents who buy a product are chosen randomly.

When agent $i$ buys a product its capital decreases by the price of product $p$ :

$$
k_{i}(t)=k_{i}(t)-P_{p} \text {. }
$$

\subsubsection{Market shock}

At this point a market cycle is finished. Due to the fact that agents of the consumption goods sector never experience unemployment (see the definition of $L_{C G}$ ) it is assumed that if the market receives a shock it will only propagate to the agents in the durable goods sector. It is also assumed that the agents "feel shocked" only for a fixed amount of time and the effect of a shock is an increase in risk aversion. The duration of a shock is different for each agent though - as it would be in a real world scenario. Every agent becomes shocked at the same time and is assumed to remain in that state for a random uniform amount of time.
After that time the agents recover from the shock instantaneously. Let $t_{s, i}$ denote the time agent $i$ suffers a shock. Denote the remaining time of the shock affecting agent $i$ by $\Delta t_{s, i}(t)$ at time $t$. If agent $i$ is shocked at time $t_{s}$, then

$t_{s, i}=t_{s}$,

$\Delta t_{s, i}\left(t_{s}\right) \sim U\left(\Delta t_{s, \text { lower }}, \Delta t_{s, \text { upper }}\right)$, and

$a_{i}\left(t_{s}\right)=\eta a_{i}\left(t_{s}-1\right)$,

where $\eta>1$ is a model parameter that characterizes the increase of risk aversion in case of a shock. In every cycle after an agent optimizes its needs the duration of shock decreases:

$\Delta t_{s, i}(t)=\Delta t_{s, i}(t-1)-1$.

The change of the utility caused by a shock is assumed to be similar to the unit step function: it does not fade away continuously, instead when the duration of the shock reaches its end the risk aversion is set back to its original value before the shock, formally

$a_{i}(t)=\left\{\begin{array}{c}\eta^{-1} a_{i}(t-1), \Delta t_{s, i}(t)=0 \\ a_{i}(t-1), \Delta t_{s, i}(t)>0\end{array}\right.$.

It is assumed that when an agent becomes unemployed it does not get shocked automatically. This assumption is based on people usually looking at unemployment as a momentary state and do not set back their spending right away.

\section{Simulation results}

We run two kinds of simulations - one without a market shock and one with a market shock. The simulation with market shock is executed with both quasilinear utility and exponential utility. As the model is stochastic in nature, each simulation is run several times (1000 iterations) and all the simulated trajectories are aggregated to guarantee a representative result. The average coefficient of variation between runs for a time step is 0.23 .

\subsection{Exponential utility}

First we show the results using an exponential utility function. The calibrated parameters for the simulations are listed in Table 1. The parameters are not meant to mirror reality exactly, only in magnitude, since emphasis is on the underlying effects. The output of the calibrated model must be analyzed to verify the model. First we examine how the model behaves without a market shock. Fig. 1 shows how 
the cumulated mean of the vehicle sales changes after each period. It can be seen that the sales converge to a steady state exponentially. This steady state should be determined by calculating the mean of the last few hundred values where the market surely reached that state - calculating the mean of the whole dataset would be biased by the outlier values of the first few hundred cycles where the market is still trying to reach a convergent state, which is 20.07. It can be seen in Fig. 1 that the simulated data oscillates symmetrically around the mean - since the mean is not a robust statistic it should be avoided in determining when a steady state is reached, but in this case the outliers even each other out, resulting in a representative mean.

In the next simulation a market shock is induced - we can see from Fig. 1 that the market has reached a steady state by $t_{s}=950$, allowing us to investigate the consequence of a shock without any other former events biasing it. Since the upper bound of the agents' shock duration is $\Delta t_{\text {upper }}=148$ we expect that the market should correct the effect of the shock and return to its original state around the $950+148=1098$ market cycle. To get a better understanding of the mean-reverting nature of the market state we apply a centered moving average with a 148 wide window on the simulated sales. The simulation results confirm the expected mean-reversion (discussed in the introduction), as it can be seen in Fig. 2. By fitting an exponential function to the part of the time series that follow the shock we found that it describes the nature of convergence very well $\left(R^{2}=0.979\right)-$ see Fig. 3. Given the results, the model output is in accordance with theoretical expectations.

Now we evaluate how the model performs against real life data. The dataset containing the auto and light truck sales in the US is available on the website of the Bureau of Economic Analysis. ${ }^{2}$ We selected the appropriate records of the monthly historical data which contain the effects of the 2008 financial crisis from July 2006 until October 2014 , and compare them with the results from our simulation that include the effects of the induced shock. In Fig. 4 we scale both datasets to the zero-one interval to make them comparable.

It can be seen that even though the historical data is much more volatile than the simulated, the two have quite similar features. Their residuals can be seen in Fig. 5 - they are normally distributed with a p-value of 0.8594 according to the Shapiro-Wilk test, and have a zero mean with p-value 0.6724 according to the t-test. By calculating the autocorrelation

2 http://bea.gov/national/xls/gap_hist.xls, Table 6. function (shown in Fig. 6) we can see that the residuals are just slightly autocorrelated, and the Wald-Wolfowitz runs test shows that the residuals are independent with a p-value of 0.1594 . Hence, the residuals do not quite come from a strict white noise process, but they are very close to it.

To reduce the increase in residuals caused by the heavy fluctuations of the historical data we smooth both the historical and the simulated dataset with a rolling mean of window-size 12, which can be seen in Fig. 7.

Table 1 Calibrated model parameters, assuming exponential utility

\begin{tabular}{lc}
\hline Parameter & Value [measurement unit] \\
\hline$L_{C G S}, L_{C D S}$ & $500[\mathrm{head}]$ \\
$P_{\text {car }}{ }^{3}$ & $26000[\$]$ \\
$P_{C G}$ & $1000[\$]$ \\
$\delta_{c a r}$ & $1 / 37$ \\
$\delta_{C G}$ & 1.0 \\
$L_{c a r}$ & $0.5[\mathrm{head}]$ \\
$\Delta k_{C G S}, \Delta k_{C D S}{ }^{4}$ & $4417[\$]$ \\
$\Delta k_{U}^{5}$ & $1000[\$]$ \\
$\alpha$ & 1.49 \\
$x_{m}$ & $87000[\$]$ \\
$T$ & $2500[\mathrm{period}]$ \\
$a_{\text {lower }}$ & $\frac{37-5}{37} \times 0.71$ \\
& \\
$a_{\text {upper }}$ & $\frac{37+5}{37} \times 0.71$ \\
$\Delta t_{\text {s,lower }}$ & $0[\mathrm{period}]$ \\
$\Delta t_{\text {s,upper }}$ & $5 * 37[\mathrm{period}]$ \\
$\eta$ & 2.0 \\
\hline
\end{tabular}

Convergence of simulated car sales to a steady state without a shock

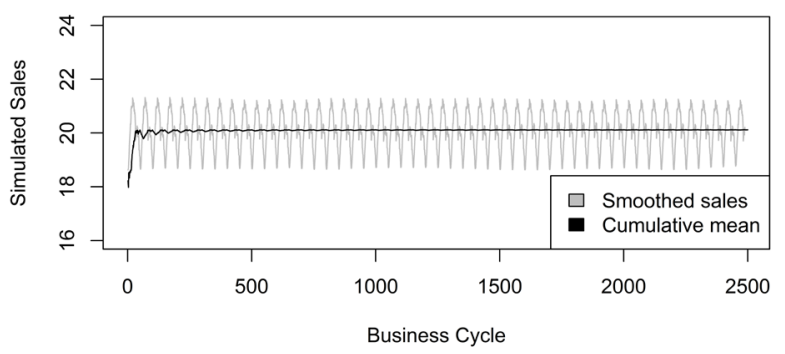

Fig. 1 Convergence of simulated car sales to a steady state if there is no market shock induced in the simulation, assuming exponential utility

3 Source: http://www.statista.com/statistics/183745/average-price-ofus-new-and-used-vehicle-sales-and-leases-since-1990/

4 Source: US Census Bureau reports on household income (http://www. census.gov/hhes/www/income/)

5 Source: Dept. of Labor and Workforce Development, State of New Jersey (http://lwd.dol.state.nj.us/labor/) 
Mean-reversion following a shock

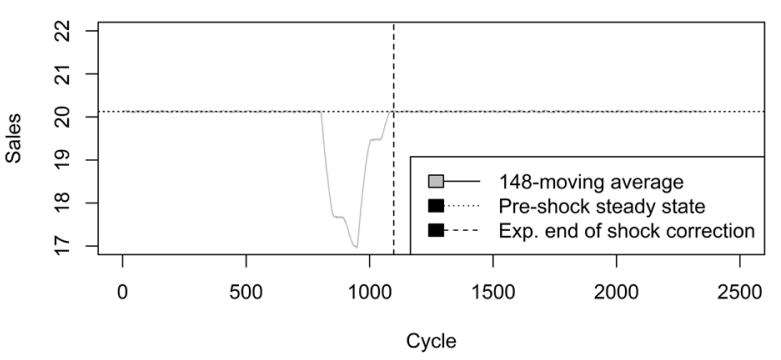

Fig. 2 Mean-reversion effect taking place after a single, unit-step-like market shock, assuming exponential utility

Exponential nature of the mean-reversion

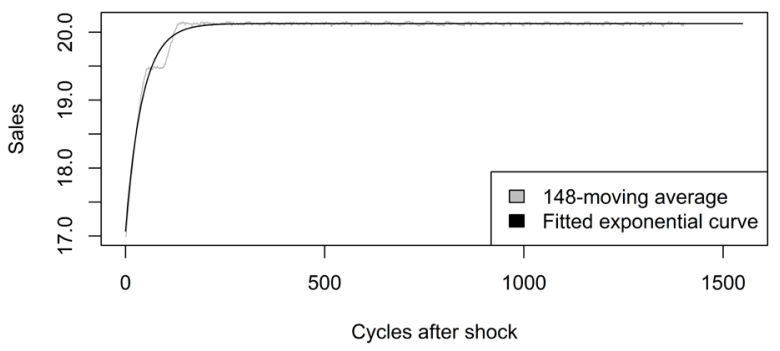

Fig. 3 Exponential nature of the mean-reversion effect after a unit-steplike shock, assuming exponential utility

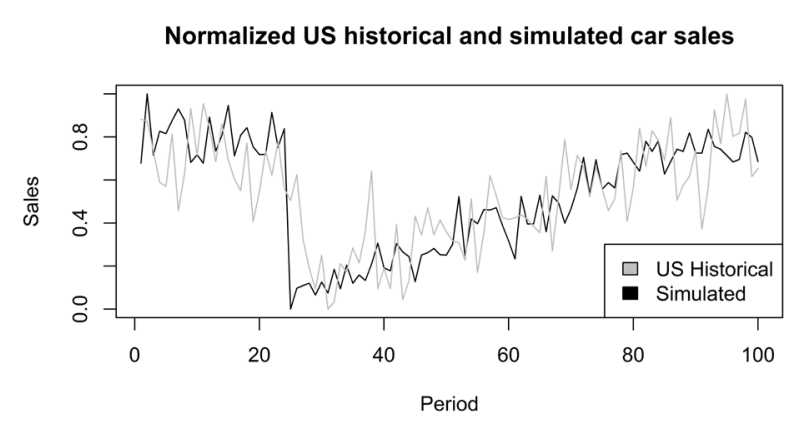

Fig. 4 US historical and simulated car sales after normalization, assuming exponential utility

Residuals of the fit

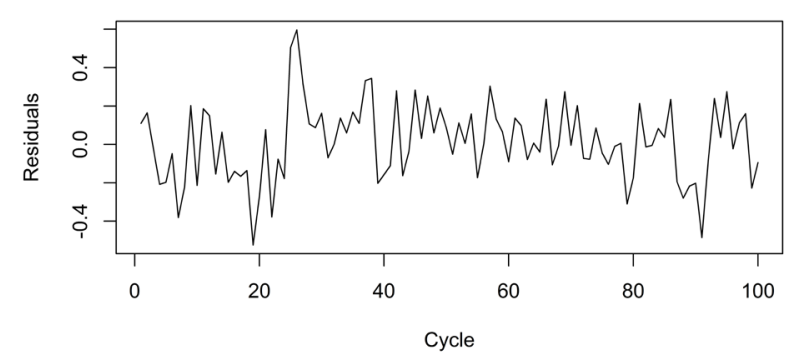

Fig. 5 Residuals after removing the simulated sales from the historical sales, assuming exponential utility

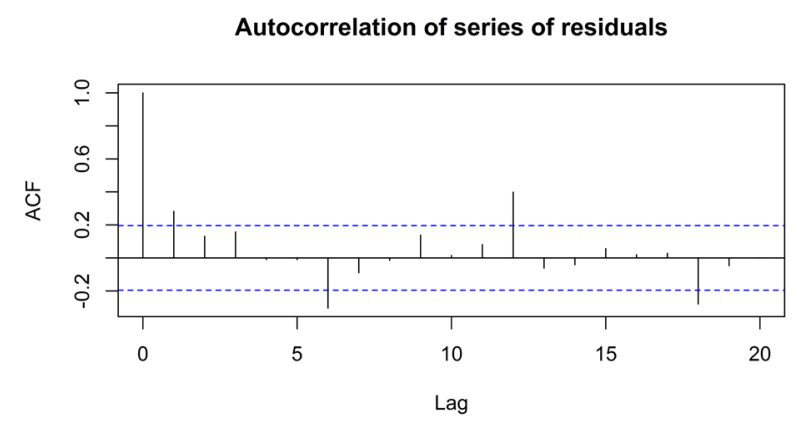

Fig. 6 Autocorrelation of model residuals, assuming exponential utility

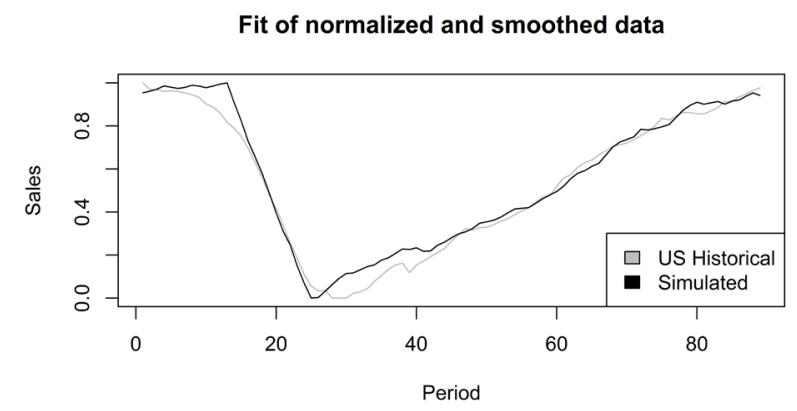

Fig. 7 Normalized simulated and US historical car sales after smoothing, assuming exponential utility

Table 2 Fit metrics of the model, assuming exponential utility

\begin{tabular}{lc}
\hline Metric & Value \\
\hline$R M S E$ & 0.0507 \\
$M A E$ & 0.0374 \\
$R^{2}$ & 0.9799 \\
\hline
\end{tabular}

To provide additional measurements of the goodness of fit we calculate the root mean squared error (RMSE), the mean absolute error (MAE) and the $R^{2}$ values on the transformed datasets, presented in Table 2. We also use the F-test to determine whether the ratio of variances of the residuals and the transformed historical data significantly differs from 1 or not. The null-hypothesis that the ratio of variances is equal to 1 has a $p$-value less than $2.2 \mathrm{e}-16$, which means it can be rejected. This shows that our model reduces the unexplained variance in the realworld data caused by an exogenous shock significantly.

\subsection{Quasilinear utility}

Next we show our results using a quasilinear utility function. The calibrated parameters for the simulations are the same as in Table 1, the only differences are shown in Table 3. 


\begin{tabular}{lc}
$\frac{\text { Table } 3 \text { Calibrated model parameters, assuming quasilinear utility }}{\text { Parameter }}$ & Value [measurement unit] \\
\hline$a_{\text {lower }}$ & $\frac{37-5}{37} \times 0.73$ \\
$a_{\text {upper }}$ & $\frac{37+5}{37} \times 0.73$ \\
\hline
\end{tabular}

Fig. 8 demonstrated that a steady state is achieved by the new model as well, while Fig. 9 and Fig. 10 show that by using a quasilinear utility function the mean-reversion is almost identical to the prior results. In this case we achieved $R^{2}=0.9783$.

Again, we compare the results of the simulation with the historical data - Fig. 11 shows the two transformed time series. The residuals (shown in Fig. 12) have zero mean with $\mathrm{p}$-value 0.5378 , are normally distributed with $\mathrm{p}$-value 0.3959 , and are independent with p-value 0.0704. They are slightly autocorrelated, as can be seen from Fig. 13. Fig. 14 shows how the simulated results fit the historical date after smoothing the heavy fluctuations. To provide additional measurements of the goodness of fit we calculate the root mean squared error (RMSE), the mean absolute error (MAE) and the $R^{2}$ values on the transformed datasets again, presented in Table 4 . The F-test returned a p-value of $1.099 \mathrm{e}-07$, thus the model significantly reduces the unexplained variance.

\section{Summary}

According to the National Bureau of Economic Research, the US economy was in recession between late 2007 and the middle of 2009. Although these dates suggest that the cycle was relatively short, the recovery has been weak for quite a while after the technical end of the recession. In our paper, we focused on one single explanation of this. Our conjecture is that in a highly reserved economy, such as the US, the cycle manifests itself predominantly in the fluctuation of consumer durables, which in turn cause the households relatively little pain. Consumers don't suffer much if they stop buying new consumer durables - such as automobiles, the example we used in this paper - because they can continue to use their "old" vehicle for quite a while without much welfare loss, highly motorizing business cycles. In the framework of a two-sector economy agent-based model, we illustrated our thesis with the fluctuations in car sales in the period 2006-2014. Subsequently we used an agent based simulation, developed in object oriented paradigm and showed that our conjectures do

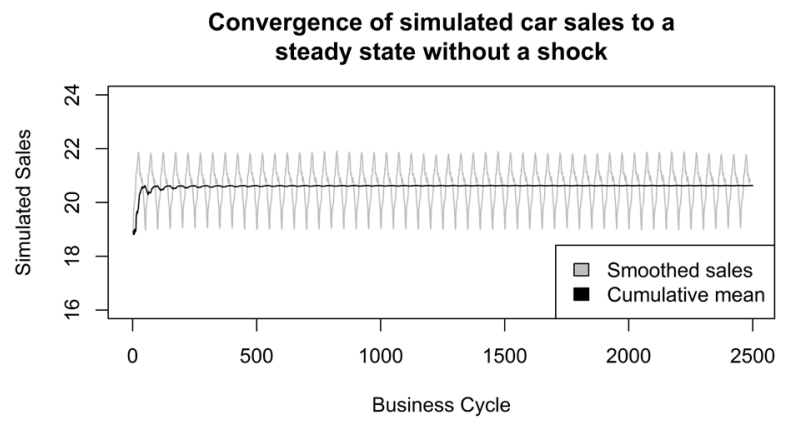

Fig. 8 Convergence of simulated car sales to a steady state if there is no market shock induced in the simulation, assuming quasilinear utility

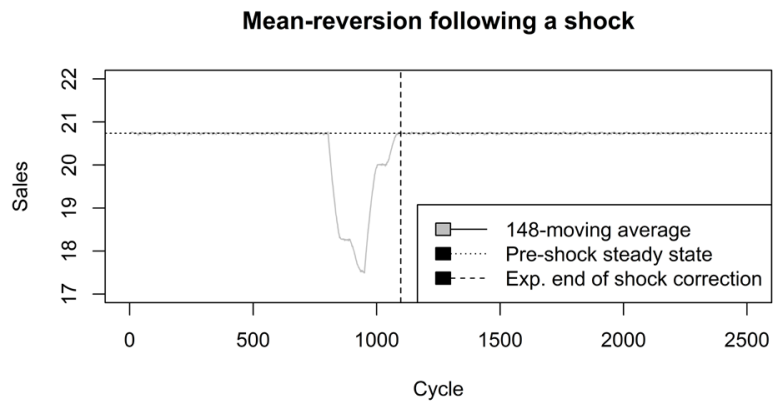

Fig. 9 Mean-reversion effect taking place after a single, unit-step-like market shock, assuming quasilinear utility

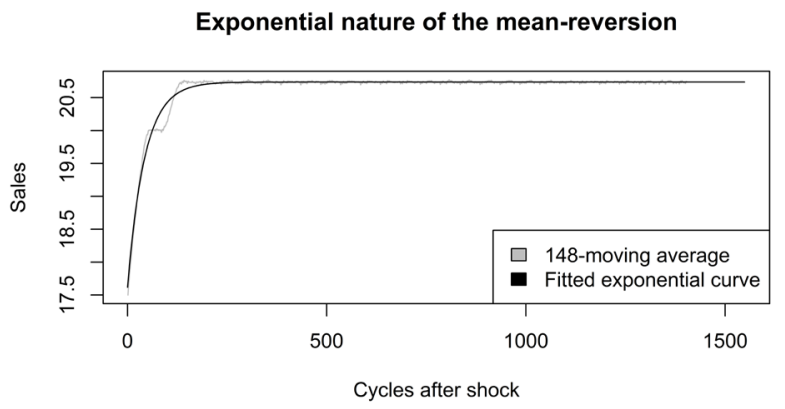

Fig. 10 Exponential nature of the mean-reversion effect after a unitstep-like shock, assuming exponential utility

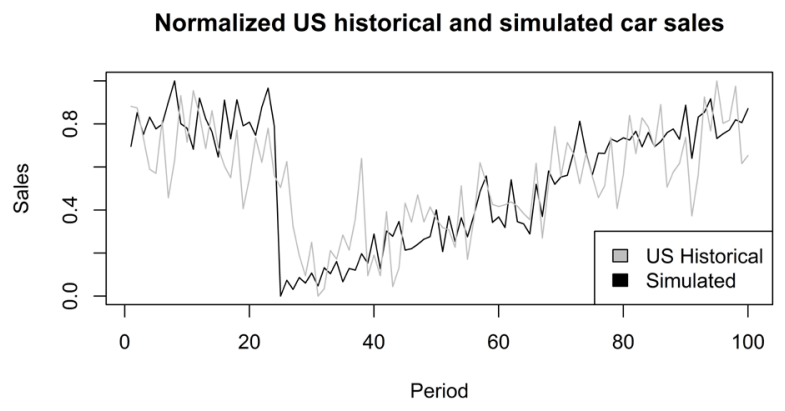

Fig. 11 US historical and simulated car sales after normalization, assuming quasilinear utility 


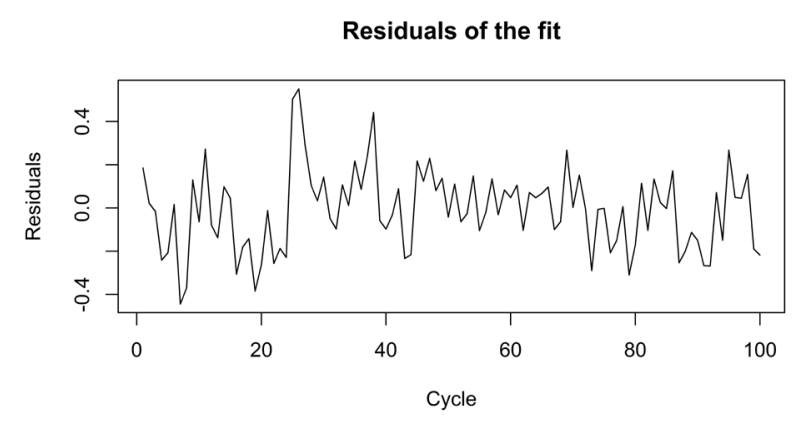

Fig. 12 Residuals after removing the simulated sales from the historical sales, assuming quasilinear utility

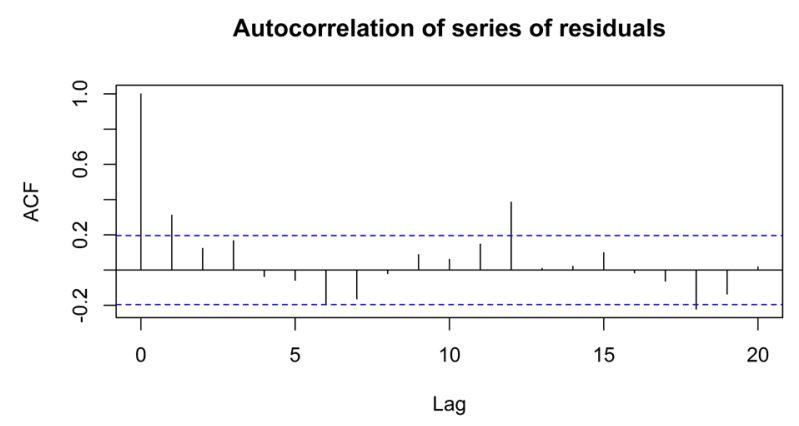

Fig. 13 Autocorrelation of model residuals, assuming exponential utility

hold if applied to real-life statistical data. We found that the market under our assumptions shows robustness to different utility functions. Our model is not a generic one that resembles all possible or historically experienced business

\section{References}

[1] Baxter, M. "Are Consumer Durables Important for Business Cycles?", The Review of Economics and Statistics, 78(1), pp. 147-155, 1996.

[2] Molnar, F. "The 1974-75 Recession in the USA: a lot of Facts and some Lessons", Acta Oeconomica, 17(2), pp. 177-202, 1976.

[3] Molnar, F. "Consumers' Investment Versus Capital Investment (A Contribution to the Theory of Contemporary Business Fluctuations)", Acta Oeconomica, 26(1-2), pp. 133-155, 1981.

[4] Berger, D., Vavra, J. "Consumption Dynamics During Recessions", NBER Working Paper Series, 20175, 2014. https://doi.org/10.3386/w20175

[5] Stolyarov, D. "Turnover of Used Durables in a Stationary Equilibrium: Are older goods traded more?", Journal of Political Economy, 110(6), pp. 1390-1413, 2002. https://doi.org/10.1086/343745

[6] Veblen, T. "The Theory of the Leisure Class", B. W. Huebsch, New York, USA, 1899.

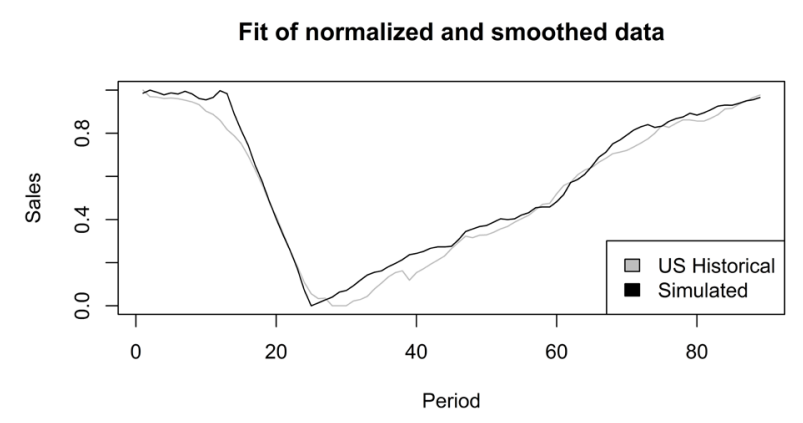

Fig. 14 Normalized simulated and US historical car sales after smoothing, assuming exponential utility

Table 4 Fit metrics of the model, assuming quasilinear utility

\begin{tabular}{lc}
\hline Metric & Value \\
\hline$R M S E$ & 0.0505 \\
$M A E$ & 0.0397 \\
$R^{2}$ & 0.9855 \\
\hline
\end{tabular}

cycles, only the present situation is in our focus. We examine one factor driven cycle in which the upward and downward changes in automobile purchase are responsible for the self-reinforcing fluctuations of the economy as a whole. We argue that the extraordinary length of the post2008 recession and the week recovery after that can, to a large extent, be explained by the fact that consumers don't suffer much when they postpone for a longer period replacing old consumer durables to new ones.

[7] Novales, A., Fernández, E., Ruíz, J. "Economic Growth: Theory and Numerical Solution Methods", Springer-Verlag Berlin Heidelberg, Berlin, Heidelberg, Germany, 2009.

[8] Prescott, E. C. "Theory Ahead of Business Cycle Measurement", Carnegie-Rochester Conference Series on Public Policy, 25, pp. 11-44, 1986.

https://doi.org/10.1016/0167-2231(86)90035-7

[9] Fiaschi, D., Lavezzi, A. M. "Nonlinear Economic Growth: Some Theory and Cross-country Evidence", Journal of Development Economics, 84(1), pp. 271-290, 2007. https://doi.org/10.1016/j.jdeveco.2005.10.007

[10] Campbell, J. Y. "Inspecting the Mechanism: An Analytical Approach to the Stochastic Growth Model", National Bureau of Economic Research Working Paper, 4188, 1992.

https://doi.org/10.3386/w4188

[11] Marsiglio, S., La Torre, D. "A Note on Demographic Shocks in a Multi-Sector Growth Model", Economics Bulletin, 32(3), pp. 2293-2299, 2012. 
[12] Bucci, A., Colapinto, C., Forster, M., La Torre, D. "Stochastic technology shocks in an extended Uzawa-Lucas model: closed-form solution and long-run dynamics", Journal of Economics, 103(1), pp. 83-99, 2011.

https://oi.org/10.1007/s00712-011-0193-0

[13] Noy, I., Nualsri, A. "What do Exogenous Shocks Tell Us about Growth Theories?", University of Hawaii Economics Working Paper 07-28, 2007.

[14] Bousquet, F., Le Page, C. "Multi-agent simulations and ecosystem management: a review", Ecological Modelling, 176(3-4), pp. 313332, 2004.

https://doi.org/10.1016/j.ecolmodel.2004.01.011

[15] Helbing, D., Balietti, S. "How to Do Agent-Based Simulations in the Future: From Modelling Social Mechanism to Emergent Phenomena and Interactive System Design", Santa Fe Institute Working Paper 11-06-024, 2011.

[16] Gatti, D.D., Gaffeo, E., Gallegati, M. "Complex agent-based macroeconomics: a manifesto for a new paradigm", Journal of Economic Interaction and Coordination, 5(2), pp. 111-135, 2010. https://doi.org/10.1007/s11403-010-0064-8

[17] Tesfatsion, L. "Introduction", Computational Economics, 18(1), pp. 1-8, 2001.

https://doi.org/10.1023/A:1013852626172

[18] Siebers, P.-O., Aickelin, U. "Introduction to Multi-Agent Simulation", In: Encyclopaedia of Decision Making and Decision Support Technologies, pp. 554-564, 2008.

https://doi.org/10.4018/9781599048437.ch062
[19] Richiardi, M., Leombruni, R., Saam, N., Sonnessa, M. "A Common Protocol for Agent-Based Social Simulation", Journal of Artificial Societies and Social Simulation, 9(1), 2006.

[20] Klass, O. S., Biham, O., Levy, M., Malcai, O., Solomon, S. "The Forbes 400 and the Pareto wealth distribution", Economic Letters, 90(2), pp. 290-295, 2006.

[21] Benito, A. "Does Job Insecurity Affect Household Consumption?", Bank of England Working Paper, 220, 2004. https://doi.org/10.2139/ssrn.641181

[22] Guiso, L., Sapienza, P., Zingales, L. "Does Culture Affect Economic Outcomes?" Journal of Economic Perspectives, 20(2), pp. $23-48,2006$. https://doi.org/10.1257/jep.20.2.23

[23] Varian, H. R. "Microeconomic Analysis", 3rd ed., W. W. Norton \& Company, New York, USA, 1992.

[24] Arrow, K. J. "Aspects of the Theory of Risk Bearing", Reprinted in: Essays in the Theory of Risk Bearing, Markham Publishing Company, Chicago, USA, 1971, pp. 90-109.

[25] Pratt, J. W. "Risk Aversion in the Small and in the Large", Econometrica, 31(1-2), pp. 122-136, 1964. https://doi.org/10.2307/1913738 